\title{
Crossed Cerebellar Diaschisis Indicates Hemodynamic Compromise in Ischemic Stroke Patients
}

\author{
Lita von Bieberstein ${ }^{1}$. Christiaan Hendrik Bas van Niftrik ${ }^{2}$. Martina Sebök ${ }^{2} \cdot$ Mohamad El Amki $^{1} \cdot$ Marco Piccirelli $^{3}$. \\ Christoph Stippich $^{3} \cdot$ Luca Regli $^{2} \cdot$ Andreas R. Luft $^{1,4} \cdot$ Jorn Fierstra $^{2} \cdot$ Susanne Wegener ${ }^{1}$ (D)
}

Received: 23 January 2020 / Revised: 21 April 2020 / Accepted: 11 May 2020 / Published online: 6 June 2020

(C) The Author(s) 2020

\begin{abstract}
Crossed cerebellar diaschisis (CCD) in internal carotid artery (ICA) stroke refers to attenuated blood flow and energy metabolism in the contralateral cerebellar hemisphere. CCD is associated with an interruption of cerebro-cerebellar tracts, but the precise mechanism is unknown. We hypothesized that in patients with ICA occlusions, CCD might indicate severe hemodynamic impairment in addition to tissue damage. Duplex sonography and clinical data from stroke patients with unilateral ICAO who underwent blood oxygen-level-dependent MRI cerebrovascular reserve (BOLD-CVR) assessment were analysed. The presence of CCD (either CCD+ or CCD-) was inferred from BOLD-CVR. We considered regions with negative BOLD-CVR signal as areas suffering from hemodynamic steal. Twenty-five patients were included (11 CCD+ and 14 CCD-). Stroke deficits on admission and at 3 months were more severe in the CCD+ group. While infarct volumes were similar, CCD+ patients had markedly larger BOLD steal volumes than $\mathrm{CCD}^{-}$patients (median [IQR] 122.2 [111] vs. 11.6 [50.6] ml; $p<0.001$ ). Furthermore, duplex revealed higher peak-systolic flow velocities in the intracranial collateral pathways. Strikingly, posterior cerebral artery (PCA)-P2 velocities strongly correlated with the National Institute of Health Stroke Scale on admission and BOLD-CVR steal volume. In patients with strokes due to ICAO, the presence of CCD indicated hemodynamic impairment with larger BOLD-defined steal volume and higher flow in the ACA/PCA collateral system. Our data support the concept of a vascular component of CCD as an indicator of hemodynamic failure in patients with ICAO.
\end{abstract}

Keywords Crossed cerebellar diaschisis $\cdot$ ICA occlusion $\cdot$ BOLD MRI $\cdot$ Cerebrovascular reserve $\cdot$ Duplex sonography

\section{Introduction}

Crossed cerebellar diaschisis (CCD) refers to attenuated cerebral blood flow (CBF) and energy metabolism of the contralateral cerebellar hemisphere after supratentorial brain injury

Lita von Bieberstein and Bas van Niftrik contributed equally to this work.

Susanne Wegener

Susanne.Wegener@usz.ch

1 Dept. of Neurology, Clinical Neuroscience Center, University Hospital Zurich and University of Zurich, Frauenklinikstrasse 26, 8091 Zurich, Switzerland

2 Dept. of Neurosurgery, Clinical Neuroscience Center, University Hospital Zurich and University of Zurich, Zürich, Switzerland

3 Dept. of Neuroradiology, Clinical Neuroscience Center, University Hospital Zurich, Zürich, Switzerland

4 cereneo Center for Neurology and Rehabilitation, Vitznau, Switzerland
[1-3]. The term "diaschisis" was introduced by Monakow in 1914 (Greek dia "in half" and schizien "to split"), epitomizing a remote loss of excitability, induced by functional disconnection of brain regions (von Monakow 1914). Detection of CCD is achieved by the use of $\mathrm{H}_{2} \mathrm{O}$ position emission tomography, computed tomography perfusion imaging, and magnetic resonance imaging techniques, e.g. dynamic susceptibility contrast, arterial spin labelling, and most recently blood oxygenation-level-dependent (BOLD) functional MRI [4-7]. Although CCD was observed in non-vascular lesions such as glioma and does not require hemodynamic compromise [8], it has been best characterized in patients with middle cerebral artery (MCA) territory stroke $[9,10]$. In patients with acute large vessel occlusion stroke affecting the MCA or internal carotid artery (ICA), the presence of CCD was shown to correlate with the size of the initial perfusion deficit [6]. However, its relationship to clinical outcome in stroke has been controversial $[4,6,11]$. We recently described CCD in patients at the sub-acute stage of symptomatic ICA occlusion 
(ICAO) using BOLD imaging of cerebrovascular reserve capacity (BOLD-CVR) [4]. ICAO may persist without clinical symptoms in patients with atherosclerotic disease, due to growth of compensatory vascular collaterals over time [12]. However, if hemodynamic impairment is not sufficiently compensated, recurrent clinical symptoms and strokes may occur. Duplex sonography parameters are useful to identify patients with ICAO whose collateral supply is insufficient and risk of recurrent ischemia higher [13, 14].

We hypothesized that in ICAO patients, in addition to stroke-induced tissue damage, hemodynamic impairment may contribute to CCD. Using duplex and BOLD-CVR, our goal was to describe a vascular component of CCD.

\section{Methods}

\section{Study Design and Cohort Description}

Patients with symptomatic ICA occlusions treated at the University Hospital Zurich Department of Neurology were prospectively collected from a BOLD-CVR database between October 2014 and October 2019. Some patient data of this cohort were previously reported $[4,5,15]$. Patients were excluded if they had a significant (medium- or high-grade) contralateral stenosis or occlusion of either the ICA or within the Circle of Willis. Patients were treated according to standard clinical practice. All patients gave written informed consent for data research and imaging procedures according to the study protocol. The study was approved by the local ethics committee (Kantonale Ethikkommission Zurich; KEK-ZH-Nr 2012-0427). In addition to routine head and neck vessel imaging and a complete duplex ultrasound of the brain-feeding arteries (extra- and intracranial, transforaminal, transorbital), patients received a BOLD MRI with a standardized carbon dioxide $\left(\mathrm{CO}_{2}\right)$ challenge to measure CVR [4]. After initial hospitalization, patients had either a clinical follow-up after 3 months or a telephone interview to determine functional independence (modified Rankin Scale, mRS). The time interval between duplex and BOLD assessment was on average 28 days (median 5, min 0, $\max 279$ days). Neurological deficits were graded by the treating stroke physicians using the National Institute of Health Stroke Scale (NIHSS). Clinical parameters, comorbidities, and risk factors were extracted from patients' clinical charts.

\section{BOLD-CVR Imaging and Determination of CCD and Steal Phenomenon}

The $\mathrm{CO}_{2}$ stimulus was administered using a computercontrolled gas blender with prospective gas targeting algorithms (RespirAct ${ }^{\mathrm{TM}}$, Thornhill Research Institute, Toronto, Canada). BOLD-CVR calculations using the combination of
BOLD fMRI and the RespirAct ${ }^{\mathrm{TM}}$ have been shown to be highly reproducible [16]. The RespirAct ${ }^{\mathrm{TM}}$ allows for precise targeting of the arterial partial pressure of oxygen and $\mathrm{CO}_{2}$ [17]. During the BOLD-CVR study, all subjects were initially clamped at their own resting $\mathrm{CO}_{2}$ value [18]. $\mathrm{CO}_{2}$ was subsequently increased using a continuous step protocol of $10 \mathrm{mmHg}$ above their resting $\mathrm{CO}_{2}$ value for $80 \mathrm{~s}$. Oxygen was maintained at a level of approximately $105 \mathrm{mmHg}$. All MRI data were acquired on a 3-T Skyra VD13 (Siemens Healthcare, Erlangen, Germany) with a 32-channel head coil. The BOLD fMRI parameters included an axial twodimensional (2D) single-shot EPI sequence planned on the anterior commissure-posterior commissure line plus $20^{\circ}$ (on a sagittal image), voxel size $3 \times 3 \times 3 \mathrm{~mm}^{3}$, acquisition matrix $64 \times 64 \times 35$ ascending interleaved slice acquisition, slice gap $0.3 \mathrm{~mm}$, GRAPPA factor 2 with 32 ref. lines, repetition time (TR)/echo time (TE) $2000 / 30 \mathrm{~ms}$, flip angle $85^{\circ}$, bandwidth $2368 \mathrm{~Hz} / \mathrm{Px}$, and field of view $192 \times 192 \mathrm{~mm}^{2}$. Two hundred volumes were acquired for the CVR study. A threedimensional (3D) T1-weighted Magnetization Prepared Rapid Acquisition Gradient Echo image was also acquired with the same orientation as the fMRI scans for overlay purposes. The acquisition parameters included voxel size $0.8 \times$ $0.8 \times 1.0 \mathrm{~mm}^{3}$ with a field of view $230 \times 230 \times 176 \mathrm{~mm}$ and scan matrix $288 \times 288 \times 176$, TR/TE/TI 2200/5.14/900 ms, and flip angle $8^{\circ}$. All BOLD fMRI volumes were preprocessed using SPM 12 (Statistical Parameter Mapping Software, Welcome Department of Imaging Neuroscience, University College of London, London, UK). Pre-processing included time and motion correction and smoothing with a Gaussian kernel of $6 \mathrm{~mm}$ full-width at half-maximum. The T1 image was aligned to the mean BOLD volume to allow the $\mathrm{T} 1$ to function as an anatomical overlay. The T1 was then segmented into grey matter, white matter, cerebrospinal fluid, skin, and skull probability maps. Only voxels with a combined probability of $>0.8$ for grey or white matter were considered for further analysis. Construction of final BOLD-CVR maps was done using in-house MATLAB R2019a scripts. To correct for difference in delay between the $\mathrm{CO}_{2}$ time series and BOLD fMRI time series on a voxel-by-voxel basis, a temporal smoothing of the BOLD fMRI signal time courses with a high-pass filter was done. Afterwards, the $\mathrm{CO}_{2}$ time series was corrected on a voxel-wise basis using a previously written and published algorithm [19], so that the increase in $\mathrm{CO}_{2}$ corresponds in time with the response of the BOLD fMRI. After this voxel-wise time correction, BOLD-CVR, defined as the $\%$ BOLD fMRI signal change/mmHg $\mathrm{CO}_{2}$, was calculated from the slope of a linear least square fit of the BOLD signal time course to the $\mathrm{CO}_{2}$ time series over the whole time series [19]. The application in stroke patients has also been described previously $[4,5]$. For each patient, individual hemispheric cerebellar masks were created with a subjects' specific subcortical anatomic allotment using Freesurfer software 

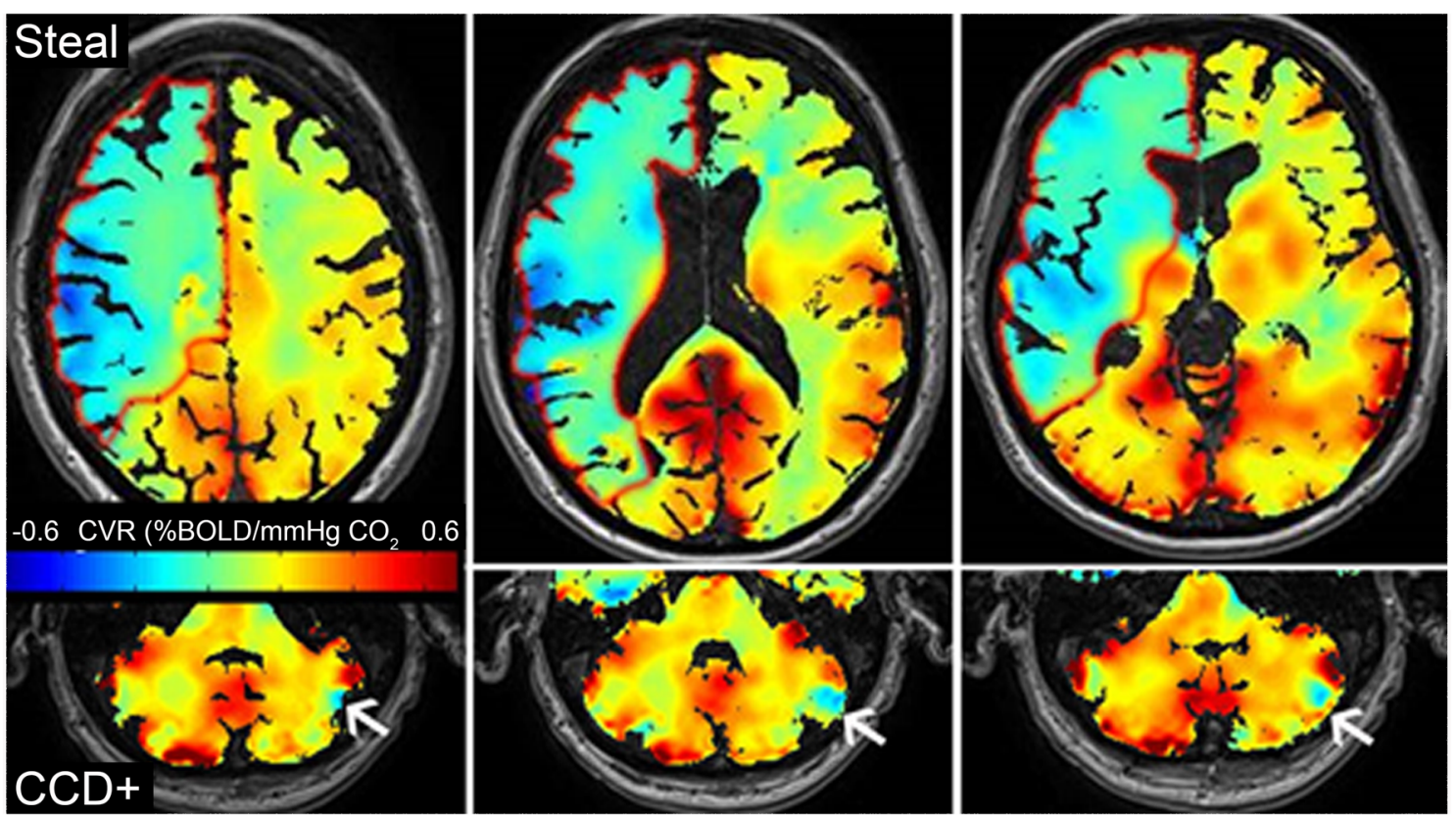

Fig. 1 BOLD-CVR assessment of CCD. Example of a patient with hemodynamic impairment in the right MCA territory due to right ICA occlusion. Three slices are shown. Steal phenomenon (i.e. negative BOLD-CVR) is depicted in green-blue and outlined in red on the upper panel. The lower panel shows three cerebellar slices of the same patient. Reduced CVR is observed on the contralateral cerebellar hemisphere (white arrow) (http://surfer.nmr.mgh.harvard.edu) [20,21]. These cerebellar masks were then used as a region of interest to determine BOLD-CVR within the whole ipsilateral cerebellar hemisphere and the whole contralateral-crossed-cerebellar hemisphere. This allowed determining the BOLD-CVR cerebellar asymmetry index (i.e. the percentage difference between both cerebellar hemispheres). Classification into CCD positive (CCD+) or CCD negative ( $\mathrm{CCD}-$ ) was then achieved using a BOLD-CVR cerebellar asymmetry index of $>6 \%$, as previously described [4]. Brain areas exhibiting steal phenomenon ipsilateral to the ICAO were determined based on negative BOLD-CVR. All voxels surrounding the anterior cranial fossa and within ventricles were excluded to reduce the number of artificially negative BOLD-CVR values. All voxels showing negative BOLD were taken and the volume of negative BOLD-CVR values was then identified by determining the volume of negative BOLD-CVR voxels on the T1weighted image (i.e. (voxel size: $0.8 \times 0.8 \times 1) \times 1000=\mathrm{ml}$ ).

\section{Duplex Imaging}

Colour-coded duplex ultrasound imaging of the extra- and intracranial (transtemporal/transorbital/transforaminal) arteries was performed on a Siemens Accuson X 2000 clinical duplex scanner according to standardized protocols within the Department of Neurology clinical routine. Vascular segments were described previously [13]. We recorded peaksystolic velocities (PSV) and end-diastolic velocities (EDV) in centimetres per second. To classify routes of collateral flow, the four main collateral pathways were identified according to $[14,22]$ as (1) anterior communicating artery (ACoaA), (2) posterior communicating artery (PCoA), (3) leptomeningeal, and (4) ophthalmic artery (OA) collaterals.

\section{Analysis of Infarct Volume}

Infarct volume was determined from diffusion-weighted MRI images (DWI) acquired in clinical routine on average 5 days (median 2, min 0, max 23) after stroke using the software Image J (NIH, version 2.0.0/rc/67/1.52 s). We outlined the hyperintense lesion slice by slice with a semi-automated thresholding tool and calculated infarct volume by multiplying pixel number with pixel size and slice thickness (+ interslice gap).

\section{Statistical Analyses}

Statistical analyses were performed using SPSS (IBM, Vs 25). For group comparisons, we used the nonparametric Fisher's exact test (categorical measurements) and 2-tailed Mann-Whitney $U$ test (continuous measurements). For bivariate correlation analyses of scales, the Pearson correlation coefficient was calculated. $p$ values $<0.05$ were considered significant. For correlations with ordinal values like stroke severity scores, the Spearman correlation coefficient was used. 
Table 1 Patient clinical characteristics

\begin{tabular}{|c|c|c|c|c|}
\hline & $\begin{array}{l}\text { All } \\
n=25(\%)\end{array}$ & $\begin{array}{l}\text { CCD+ } \\
n=11(\%)\end{array}$ & $\begin{array}{l}\mathrm{CCD}- \\
n=14(\%)\end{array}$ & $p$ value \\
\hline \multicolumn{5}{|l|}{ Demographic data } \\
\hline Age (range) & $65(33-81)$ & $66(49-77)$ & $64(33-81)$ & 0.979 \\
\hline Female & $6(24)$ & $3(27.3)$ & $3(21.4)$ & 1 \\
\hline \multicolumn{5}{|l|}{ Medical history, $n(\%)$} \\
\hline Smoking & $15(60)$ & $9(81.8)$ & $6(42.9)$ & 0.099 \\
\hline Hypertension & $20(80)$ & $10(90.9)$ & $10(71.4)$ & 0.341 \\
\hline Diabetes & $5(20)$ & $3(27.3)$ & $2(14.3)$ & 0.623 \\
\hline CAD & $8(32)$ & $3(27.3)$ & $5(35.7)$ & 0.695 \\
\hline pAOD & $4(16)$ & $2(18.2)$ & $2(14.3)$ & 1 \\
\hline Dyslipidemia & $17(68)$ & $7(63.6)$ & $10(71.4)$ & 1 \\
\hline Atrial fibrillation & $3(12)$ & $2(18.2)$ & $1(7.1)$ & 0.565 \\
\hline \multicolumn{5}{|c|}{ Clinical scores, median (IQR) } \\
\hline NIHSS on admission & $3(5)$ & $6(8)$ & $0(3)$ & $0.005 *$ \\
\hline NIHSS at 3 months & $0(3)$ & $1(1)$ & $0(2)$ & $0.023 *$ \\
\hline $\mathrm{mRS}$ on admission & $2(3)$ & $3(2)$ & $0(3)$ & $0.031 *$ \\
\hline $\mathrm{mRS}$ at 3 months & $1(1)$ & $1(1)$ & $0(1)$ & $0.059 *$ \\
\hline \multicolumn{5}{|l|}{ TOAST, subtype, $n(\%)$} \\
\hline Large vessel disease & $15(60)$ & $6(54.5)$ & $9(64.2)$ & 0.856 \\
\hline Cardio embolic & $0(0)$ & $0(0)$ & $0(0)$ & \\
\hline Small artery disease & $0(0)$ & $0(0)$ & $0(0)$ & \\
\hline Other causes & $5(20)$ & $3(27.3)$ & $2(14.3)$ & \\
\hline Undetermined & $5(20)$ & $2(18.2)$ & $3(21.4)$ & \\
\hline
\end{tabular}

Clinical characteristics of all 25 - patients (all) and patient groups without $\left(\mathrm{CCD}^{-}\right)$or with $(\mathrm{CCD}+)$ crossed cerebellar diaschisis. $p$ values $<0.05$ in Mann-Whitney $U$ test or Fisher exact test are marked with asterisk. CAD coronary artery disease, $p A O D$ peripheral artery occlusive disease. Numbers $(n)$ and percentage or median and interquartile range (IQR) are shown

\section{Results}

Of 39 patients with symptomatic ICAO initially identified in the database, seven were excluded because of contralateral ICA stenosis or additional intracranial stenosis within the Circle of Willis. Furthermore, three patients were excluded due to insufficient or missing imaging or duplex data, and three due to uncertain symptom onset. Of the remaining 25 patients, 11 were classified CCD+ and $14 \mathrm{CCD}^{-}$. A typical BOLD-CVR image of a CCD+ patient is shown in Fig. 1.

Clinical characteristics of CCD+ and CCD- groups are summarized in Table 1. Furthermore, characteristic MRI (DWI, FLAIR, BOLD-CVR) and direct stroke corticospinal tract involvement (CST) for each patient are shown in Table 2. Sex, age, risk factors, and stroke etiologies were similar in both groups. Patients in the $\mathrm{CCD}+$ group had a significantly higher NIHSS and mRS on admission (CCD+ vs. CCD-: median [IQR]: NIHSS 6 [8] vs. 0 [3], $p=0.005$; mRS 3 [2] vs. 0 [3], $p=0.031$ ). Neurological deficit at 3 months was also more severe in the CCD+ group (NIHSS 3 months CCD+ vs. CCD - : median [IQR] 1 [1] vs. 0 [2], $p=0.023$ ), while the difference in $\mathrm{mRS}$ at 3 months was not significant (CCD+ vs. CCD-: median [IQR] 1 [1] vs. 0 [1], $p=0.059$ ). Timing of BOLDCVR imaging in relation to clinical symptoms of ischemia was variable, since this technique is only used in selected stroke patients in our center. However, the distance between symptoms and BOLD was not different in CCD+ or CCD- patients (median [IQR] 7 [61] vs. 5 [23.75] days, $p=0.803$ ). Infarcts involving the corticospinal tract (either on DWI or on FLAIR images, see Table 2) were not more frequent in patients with $\operatorname{CCD}(p=$ 0.265 ).

Duplex and BOLD parameters, displayed in Table 3, demonstrate that there were increased flow velocities in $\mathrm{CCD}+$ patients in the basilar artery (BA), contralateral PCA P1 and P2 segments, ipsilateral P2 segment, and anterior cerebral artery (ACA). Median CCD+ flow velocities in the ipsilateral PCA P1 segment were higher as well, but this trend did not reach significance. MRI determined infarct volumes were small and similar in both patients' groups. As expected, supratentorial BOLD-CVR was lower at the side of ICA occlusion compared with the contralateral side in all included patients. However, although the magnitude of hemispheric BOLD-CVR reduction was similar in CCD+ and CCD- patients, the most striking finding from BOLD imaging data was a markedly larger steal volume on the ipsilateral supratentorial hemisphere in CCD+ patients (median [IQR]: CCD+ 122.2 [111] vs. CCD- $11.6[50.6] \mathrm{ml} ; p<0.001)$.

In a next step, we searched for a correlation between individual duplex and BOLD-CVR parameters. We found a strong linear correlation between volume of steal phenomenon and flow velocity in the P2 segment of both PCAs (Pearson's $P$ for ipsilateral PCA-P2 $0.787, p<0.001$, for contralateral PCA-P2 0.753, $p<0.001$; shown for the ipsilateral P2 in Fig. 2a), as well as between steal volume and severity of initial neurological deficits on admission NIHSS (Spearman's rho 0.64, $p<0.001$ : Fig. 2b).

\section{Discussion}

In its original description, CCD was regarded a neurophysiological phenomenon, caused by disconnection of corticoponto-cerebellar tracts with deactivation of cerebellar Purkinje cells [23-25]. Gold and Lauritzen were able to elicit this neuronal deactivation by experimental ischemia as well as functional ablation of the cerebral cortex in rats, and provided the direct link to concordant decreases in cerebellar blood flow along with the depression in neural activity [26].

However, for a long time, the relevance of CCD for clinical practice was uncertain, because no clear clinical correlate of disconnection was found in stroke patients. Recently, a wider definition of diaschisis was suggested, accounting for different 
Table 2 Patient imaging findings

\begin{tabular}{|c|c|c|c|c|c|c|c|c|c|}
\hline $\begin{array}{c}\text { PAT } \\
\text { ID }\end{array}$ & Age & Sex & Event & CST & DWI & FLAIR & BOLD & $\begin{array}{l}\text { Steal } \\
\left(\mathrm{cm}^{3}\right)\end{array}$ & CCD \\
\hline 1 & 72 & $\mathrm{M}$ & $\begin{array}{l}\text { TIA/ } \\
\text { RAO }\end{array}$ & No & & & & 55.71 & No \\
\hline 2 & 71 & M & $\begin{array}{l}\text { Stroke/ } \\
\text { RAO }\end{array}$ & Yes & & & & 0 & No \\
\hline 3 & 35 & $\mathrm{~F}$ & $\begin{array}{c}\text { Stenosis } \\
\text { (Dissection) }\end{array}$ & No & & & & 0 & No \\
\hline 4 & 52 & M & Stroke & Yes & & & & 0 & No \\
\hline 5 & 76 & M & Stroke & Yes & & & & 176.28 & Yes \\
\hline 6 & 79 & $\mathrm{~F}$ & Stroke & Yes & & & & 90.47 & Yes \\
\hline 7 & 70 & $\mathrm{~F}$ & Stroke & No & & & & 10.23 & No \\
\hline 8 & 69 & M & Stroke & Yes & & & & 65.67 & Yes \\
\hline 9 & 50 & M & Stroke & No & & & & 43.52 & Yes \\
\hline 10 & 53 & M & Stroke & Yes & & & & 161.68 & Yes \\
\hline 11 & 49 & M & Stroke & Yes & & & & 122.24 & Yes \\
\hline
\end{tabular}


Table 2 (continued)

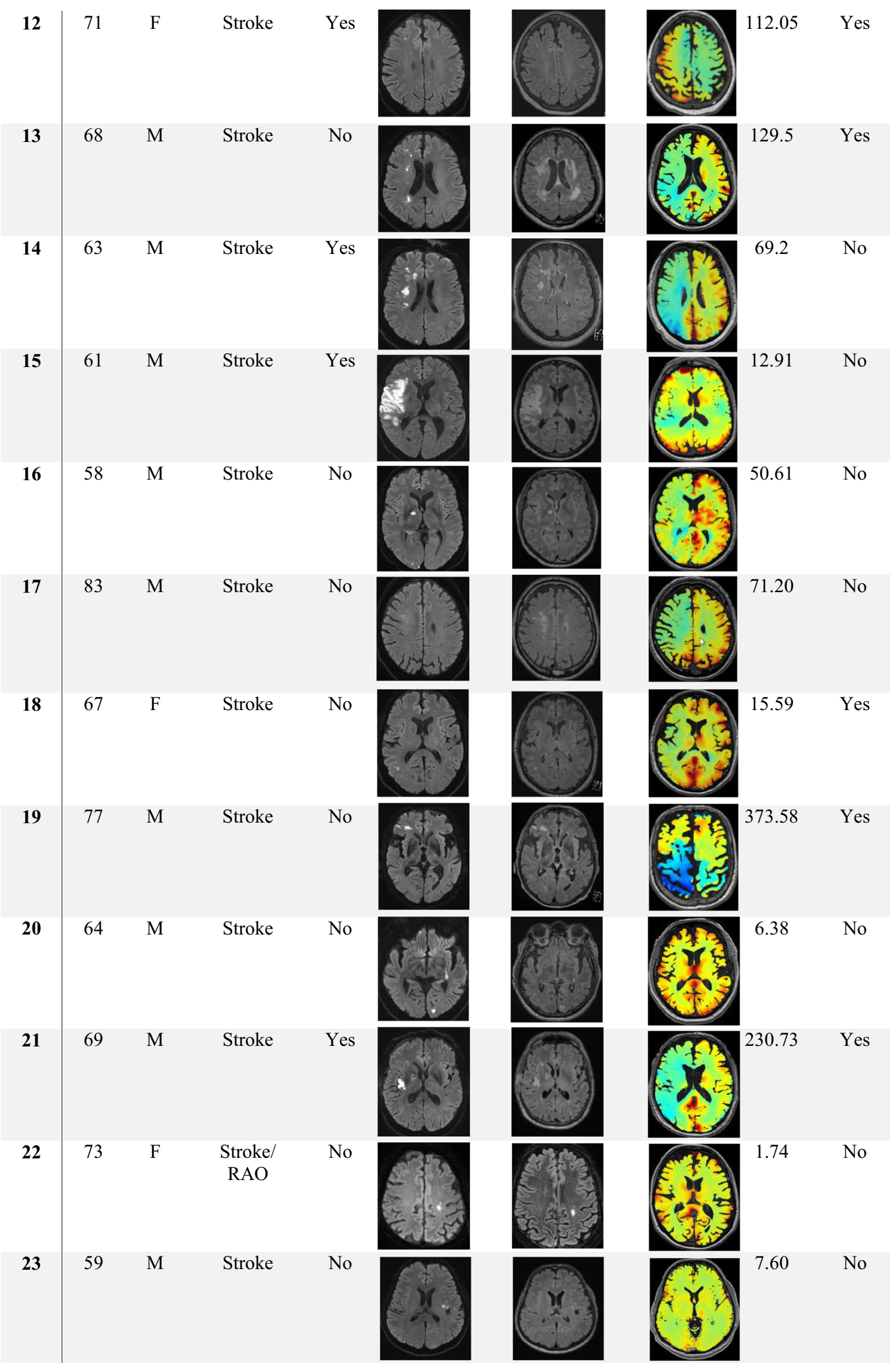


Table 2 (continued)

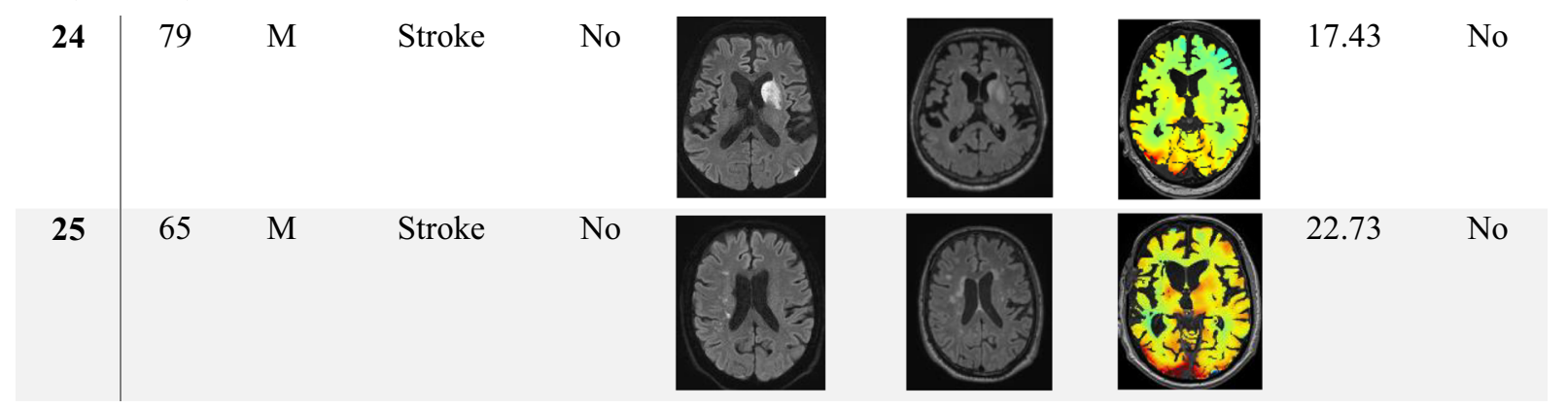

Individual patients imaging findings. Columns from left to right show age (at time of stroke); sex ( $\mathrm{F}=$ female, $\mathrm{M}=$ male); event type: stroke, transient ischemic attack (TIA), retinal artery occlusion (RAO); CST: corticospinal tract involvement on either DWI or fluid-attenuated MRI (FLAIR) images; characteristic slice of lesion on DWI, FLAIR, and BOLD-CVR; steal volume from BOLD-CVR $\left(\mathrm{cm}^{3}\right)$; and CCD+ yes/no

Table 3 Duplex and BOLD parameters

\begin{tabular}{|c|c|c|c|c|}
\hline Duplex parameter (in $\mathrm{cm} / \mathrm{s}$ ) & $\begin{array}{l}\text { All } \\
N=25\end{array}$ & $\begin{array}{l}\text { CCD+ } \\
N=11\end{array}$ & $\begin{array}{l}\text { CCD- } \\
N=14\end{array}$ & $p$ value \\
\hline \multicolumn{5}{|l|}{ Intracranial } \\
\hline PSV ACA ipsi & $67(45 ; 25)$ & $62(40 ; 11)$ & $79(57 ; 14)$ & 0.819 \\
\hline EDV ACA ips & $32(32 ; 25)$ & $26(11 ; 11)$ & $34(41 ; 14)$ & 0.757 \\
\hline PSV ACA contra & $108(86 ; 23)$ & $121(102 ; 9)$ & $97(70 ; 14)$ & 0.128 \\
\hline EDV ACA contra & $47(45 ; 23)$ & $59(49 ; 9)$ & $40.5(38 ; 14)$ & $0.015^{*}$ \\
\hline PSV M1 ipsi & $79.5(52 ; 24)$ & $73(44 ; 11)$ & $88(50 ; 13)$ & 0.234 \\
\hline EDV M1 ipsi & $37(21 ; 24)$ & $32(12 ; 11)$ & $41(35 ; 13)$ & 0485 \\
\hline PSV M1 contra & $115(37 ; 25)$ & $119(49 ; 11)$ & $114.5(38 ; 14)$ & 0.600 \\
\hline EDV M1 contra & $45(20 ; 25)$ & $47(20 ; 11)$ & $40.5(20 ; 14)$ & 0.161 \\
\hline PSV P1 ipsi & $74(52 ; 25)$ & $84(44 ; 11)$ & $73(72 ; 14)$ & 0.676 \\
\hline EDV P1 ipsi & $30(23 ; 25)$ & $34(21 ; 11)$ & $28.5(29 ; 14)$ & 0.491 \\
\hline PSV P1 contra & $69.5(31 ; 24)$ & $81.5(40 ; 10)$ & $56(32 ; 14)$ & $0.003 *$ \\
\hline EDV P1 contra & $27(14 ; 24)$ & $30(13 ; 10)$ & $20.5(16 ; 14)$ & $0.027 *$ \\
\hline PSV P2 ipsi & $66(29 ; 25)$ & $76(33 ; 11)$ & $51(15 ; 14)$ & $<0.001 *$ \\
\hline EDV P2 ipsi & $27(13 ; 25)$ & $37(17 ; 11)$ & $24(8 ; 14)$ & $0.006^{*}$ \\
\hline PSV P2 contra & $59(33 ; 25)$ & $74(22 ; 11)$ & $46(19 ; 14)$ & $0.001 *$ \\
\hline EDV P2 contra & $20(15 ; 25)$ & $24(19 ; 11)$ & $18(9 ; 14)$ & $0.027 *$ \\
\hline \multicolumn{5}{|l|}{ Transforaminal } \\
\hline PSV BA & $66(38 ; 25)$ & $79(39 ; 11)$ & $55.5(25 ; 14)$ & $0.043^{*}$ \\
\hline EDV BA & $26(11 ; 25)$ & $26(19 ; 11)$ & $25.5(11 ; 14)$ & 0.474 \\
\hline \multicolumn{5}{|l|}{ MRI parameter } \\
\hline Infarct volume $\left(\mathrm{cm}^{3}\right)$ & $3(8.6 ; 25)$ & $3.7(11.9 ; 11)$ & $1.3(8.5 ; 14)$ & 0.105 \\
\hline CST involvement $(n, \%)$ & $13(52)$ & $7(63.6)$ & $6(42.9)$ & 0.265 \\
\hline CVR supra ipsi & $0.08(0.11 ; 25)$ & $0.04(0.09 ; 11)$ & $0.09(0.06 ; 14)$ & 0.095 \\
\hline CVR supra contra & $0.14(0.08 ; 25)$ & $0.14(0.09 ; 11)$ & $0.16(0.09 ; 14)$ & 0.609 \\
\hline CVR cerebellar ipsi & $0.17(0.09 ; 25)$ & $0.18(0.17 ; 11)$ & $0.16(0.07 ; 14)$ & 0.472 \\
\hline CVR cerebellar contra & $0.16(0.08 ; 25)$ & $0.14(0.18 ; 11)$ & $0.16(0.06 ; 14)$ & 0.785 \\
\hline Steal volume $\left(\mathrm{cm}^{3}\right)$ & $50.6(108 ; 25)$ & $122.2(111 ; 11)$ & $11.6(50.6 ; 14)$ & $<0.001 *$ \\
\hline
\end{tabular}

Duplex assessment of flow (PSV/EDV) or BOLD MRI assessment (for CVR) measured ipsi—or contralateral to ICAO ("ipsi" vs. "contra") in all patients (all) and patients' groups with or without CCD. $p$ values $<0.05$ in MannWhitney $U$ test are marked with asterisk. Median (IQR; $n$ ) is shown 
Fig. 2 Correlation of hemodynamic and clinical parameters in ICA occlusion patients. Scatter plots of bivariate correlation analyses between ipsilateral BOLD-CVR-steal volume $(\mathrm{ml})$ on the $x$-axis, and a flow in the P2 segment of the ipsilateral PCA $(\mathrm{cm} / \mathrm{s})$ as well as b NIHSS on admission. Results of bivariate correlation analyses from all patients show a significant linear correlation
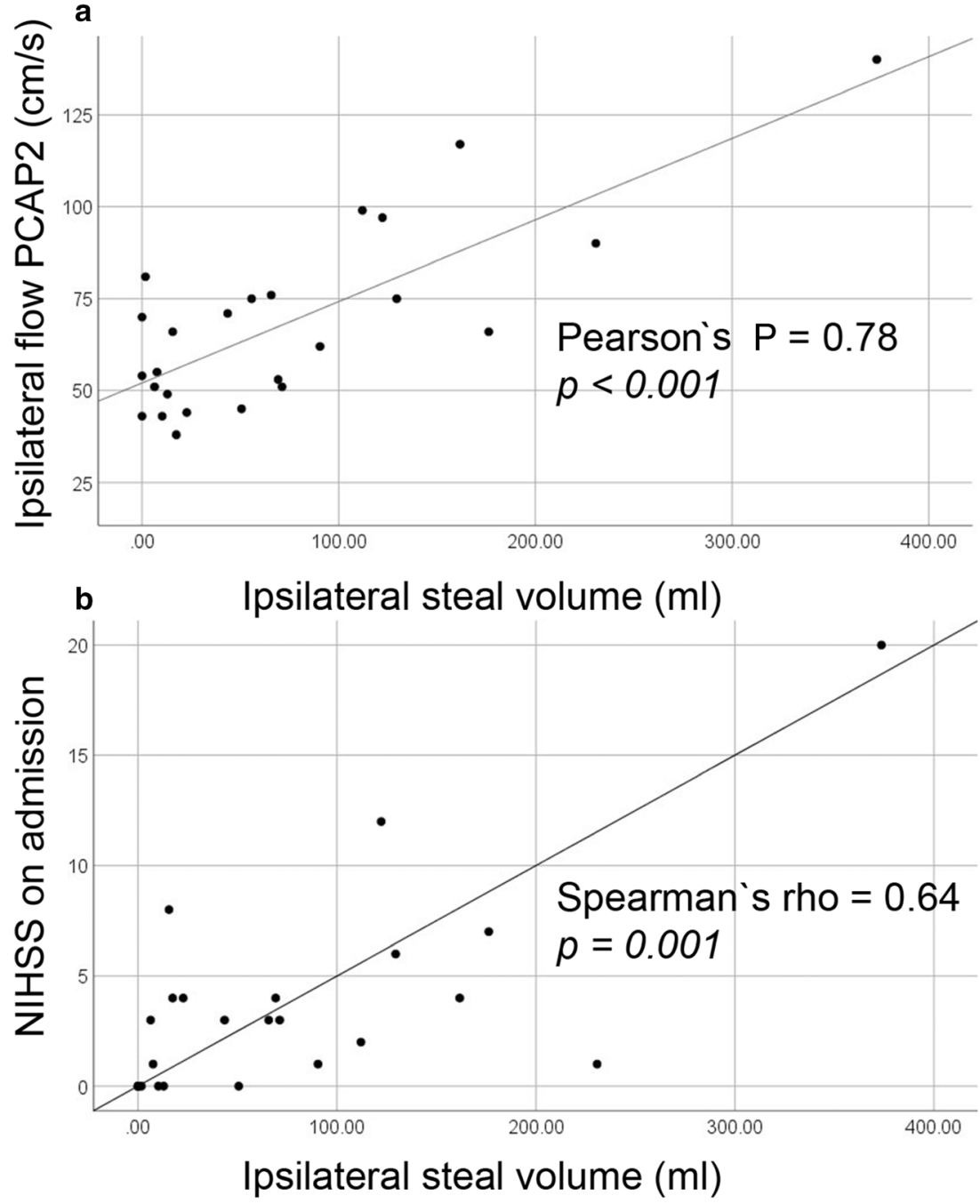

aspects of remote brain dysfunction after a lesion: focal versus non-focal (connectional or connectomal) [27]. CCD as it occurs in supratentorial stroke would be a focal type of diaschisis, but it is likely that other types of network alterations occur in focal stroke as well.

Previous work suggested that patients with MCA territory stroke and CCD had more severe supratentorial hypoperfusion and hemodynamic impairment $[4,6,7,10]$. We hypothesized that in these patients, CCD might have a potential vascular component, incorporating compensatory blood flow responses to persisting vascular occlusions affecting the MCA territory.

In this study, we demonstrate that indeed the brain area affected by severe hemodynamic compromise (i.e. steal phenomenon) was by far larger in CCD+ patients (median BOLDCVR steal volume of approximately $122 \mathrm{vs} .12 \mathrm{ml}$ ). This went along with a more severe neurological deficit on admission (median NIHSS 6 vs. 0) and at 3 months (median NIHSS 1 vs. 0). Furthermore, we were able to reveal increased hemodynamic efforts in $\mathrm{CCD}+$ patients by routine duplex: flow in collateral pathways was higher, particularly in those collateral routes coming from the posterior circulation (BA/PCA). This is similar to our findings in patients with ICA occlusion that were at higher risk of recurrent ischemia [13], where flow in bilateral PCA-P2 segments was higher. In our cohort, flow in the PCA on both sides correlated to supratentorial steal volume on the side of ICAO. Supratentorial steal volume also correlated with initial neurological deficit on admission (NIHSS). Based on our findings, we suggest that in patients with ICA occlusion, CCD+ shows a propensity towards more hemodynamic compromise and compensatory collateral flow via the Circle of Willis (CoW; Fig. 3). It is unlikely that these PCA flow increases cause the observed decrease in blood flow or BOLD signal within the contralateral cerebellar hemisphere in CCD+. This was confirmed by our BOLD data, revealing no overall differences in hemispheric cerebellar BOLD-CVR, except for the cerebellar asymmetry index.

One limitation of our study is the small sample size $(n=25)$. Furthermore, the time interval between stroke and BOLD imaging, albeit not different between $\mathrm{CCD}+/ \mathrm{CCD}$ - groups, was highly variable. Of note, patients in this study were not consecutively 


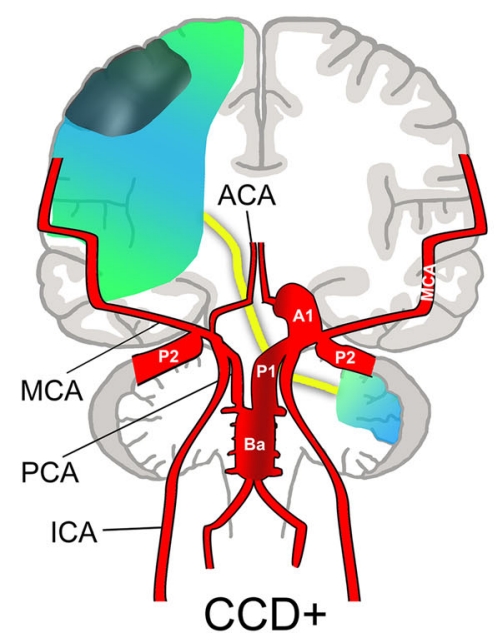

Fig. 3 Concept for a vascular component in CCD. In CCD+ patients, even with a small stroke that may have a similar volume to $\mathrm{CCD}$ patients (dark green area), hemodynamic impairment results in a large BOLD-defined ipsilateral steal volume (light blue/green area). In order to compensate hemodynamic impairment in $\mathrm{CCD}+$ patients, collateral

enrolled stroke patients with ICA occlusion, but selected patients without M1 or other intracranial artery occlusions that were able and willing to participate in additional BOLD-CVR imaging. Therefore, there was a selection bias towards less severely affected patients. Another limitation is that information about initial perfusion/mismatch status was missing for many patients, so that hemodynamic characterization of the hyper-acute stroke phase was not possible. However, while different hemodynamic factors such as the extent of collaterals may shape the severity of the initial stroke, our findings indicate that hemodynamic impairment persists beyond the hyper-acute phase of stroke. Infarct size is not an indicator of hemodynamic compromise. In contrast, BOLD-CVR imaging and duplex can help to identify patients with $\mathrm{CCD}$ and lingering deficits in cerebrovascular reserve.

\section{Summary}

We suggest that ipsilateral supratentorial hemodynamic impairment due to large vessel occlusion in CCD+ patients elicits complex neurovascular reactions leading to distant $\mathrm{CBF}$ changes. Therefore, despite a similar amount of supratentorial strokeinduced tissue damage, $\mathrm{CCD}+$ patients with ICAO had more severe hemodynamic impairment, reflected by larger supratentorial steal volumes in BOLD-CVR and increased compensatory collateral flow in the posterior circulation. This suggests a vascular component of CCD. Furthermore, CCD+ patients had more severe clinical symptoms on admission and after 3 months, indicating that severe hemodynamic impairment may induce more severe clinical deficits leading to less favourable functional recovery. Radiological infarct size estimates may therefore not capture the hemodynamic source of clinical deficits (clinical/radiological mismatch). Our findings support the use of

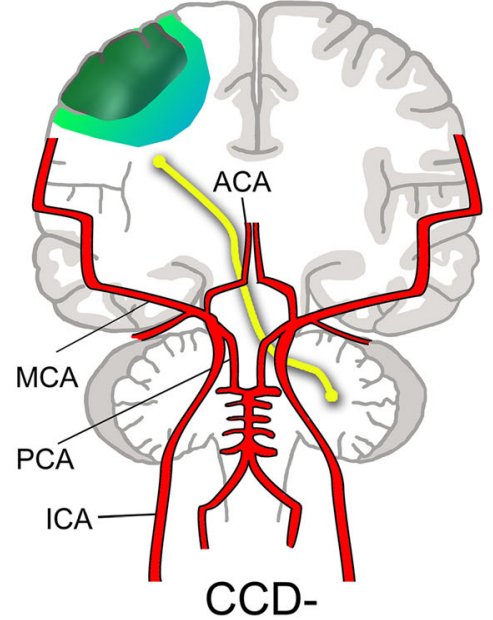

efforts are strongly increased (broadened vessel diameters in CCD+). This manifests as increased flow within the contralateral ACA (A1), supplying the retrograde ipsilateral ACA, as well as the vertebrobasilar system (BA, P1, P2)

non-invasive hemodynamic and cerebral blood flow measurements to characterize stroke patients with persisting vascular occlusion to detect impaired hemodynamic capacity. Future studies with serial BOLD-CVR measurements in patients with vascular disease may reveal if CCD changes over time in a way that is related to the clinical course.

Funding Information This project was funded by the Clinical Research Priority Program of the University of Zurich (UZH CRPP Stroke), the Swiss National Science Foundation (PP00P3 170683), the Zurich University (Forschungskredit Postdoc 2016, FK-16-040) and the Swiss Cancer League (KFS-3975-082016-R).

\section{Compliance with Ethical Standards}

Conflict of Interest The authors declare that they have no conflict of interest.

Ethical Approval All procedures performed in studies involving human participants were in accordance with the ethical standards of the institutional and/or national research committee and with the 1964 Helsinki declaration and its later amendments or comparable ethical standards. Informed consent was obtained from all individual participants included in the study.

Open Access This article is licensed under a Creative Commons Attribution 4.0 International License, which permits use, sharing, adaptation, distribution and reproduction in any medium or format, as long as you give appropriate credit to the original author(s) and the source, provide a link to the Creative Commons licence, and indicate if changes were made. The images or other third party material in this article are included in the article's Creative Commons licence, unless indicated otherwise in a credit line to the material. If material is not included in the article's Creative Commons licence and your intended use is not permitted by statutory regulation or exceeds the permitted use, you will need to obtain permission directly from the copyright holder. To view a copy of this licence, visit http://creativecommons.org/licenses/by/4.0/. 


\section{References}

1. Baron JC, Bousser MG, Comar D, Castaigne P. "Crossed cerebellar diaschisis" in human supratentorial brain infarction. Trans Am Neurol Assoc. 1981;105:459-61.

2. Baron JC, Rougemont D, Soussaline F, Bustany P, Crouzel C, Bousser MG, et al. Local interrelationships of cerebral oxygen consumption and glucose utilization in normal subjects and in ischemic stroke patients: a positron tomography study. J Cereb Blood Flow Metab. 1984;4(2):140-9.

3. Yamauchi H, Fukuyama H, Kimura J. Hemodynamic and metabolic changes in crossed cerebellar hypoperfusion. Stroke. 1992;23(6): 855-60.

4. Sebok M, van Niftrik CHB, Piccirelli M, Bozinov O, Wegener S, Esposito G, et al. BOLD cerebrovascular reactivity as a novel marker for crossed cerebellar diaschisis. Neurology. 2018;91(14): e1328-e37.

5. Fierstra J, van Niftrik C, Warnock G, Wegener S, Piccirelli M, Pangalu A, et al. Staging hemodynamic failure with blood oxygen-level-dependent functional magnetic resonance imaging cerebrovascular reactivity: a comparison versus gold standard ((15)O-)H2O-positron emission tomography. Stroke. 2018;49(3): 621-9.

6. Kunz WG, Sommer WH, Hohne C, Fabritius MP, Schuler F, Dorn F, et al. Crossed cerebellar diaschisis in acute ischemic stroke: impact on morphologic and functional outcome. J Cereb Blood Flow Metab. 2017;37(11):3615-24.

7. Strother MK, Buckingham C, Faraco CC, Arteaga DF, Lu P, Xu Y, et al. Crossed cerebellar diaschisis after stroke identified noninvasively with cerebral blood flow-weighted arterial spin labeling MRI. Eur J Radiol. 2016;85(1):136-42.

8. Liu X, Li J, Xu Q, Mantini D, Wang P, Xie Y, et al. Pathological factors contributing to crossed cerebellar diaschisis in cerebral gliomas: a study combining perfusion, diffusion, and structural MR imaging. Neuroradiology. 2018;60(6):643-50.

9. Sobesky J, Thiel A, Ghaemi M, Hilker RH, Rudolf J, Jacobs AH, et al. Crossed cerebellar diaschisis in acute human stroke: a PET study of serial changes and response to supratentorial reperfusion. $\mathrm{J}$ Cereb Blood Flow Metab. 2005;25(12):1685-91.

10. Sommer WH, Bollwein C, Thierfelder KM, Baumann A, Janssen $\mathrm{H}$, Ertl-Wagner B, et al. Crossed cerebellar diaschisis in patients with acute middle cerebral artery infarction: occurrence and perfusion characteristics. J Cereb Blood Flow Metab. 2016;36(4):74354.

11. Laloux P, Richelle F, Jamart J, De Coster P, Laterre C. Comparative correlations of HMPAO SPECT indices, neurological score, and stroke subtypes with clinical outcome in acute carotid infarcts. Stroke. 1995;26(5):816-21.

12. Hartkamp NS, Petersen ET, Chappell MA, Okell TW, Uyttenboogaart M, Zeebregts CJ, et al. Relationship between haemodynamic impairment and collateral blood flow in carotid artery disease. J Cereb Blood Flow Metab. 2018;38(11):2021-32.

13. Schneider J, Sick B, Luft AR, Wegener S. Ultrasound and clinical predictors of recurrent ischemia in symptomatic internal carotid artery occlusion. Stroke. 2015;46(11):3274-6.
14. Reinhard M, Muller T, Guschlbauer B, Timmer J, Hetzel A. Dynamic cerebral autoregulation and collateral flow patterns in patients with severe carotid stenosis or occlusion. Ultrasound Med Biol. 2003;29(8):1105-13.

15. Hendrik Bas van Niftrik C, Sebok M, Muscas G, Piccirelli M, Serra C, Krayenbuhl N, et al. Characterizing ipsilateral thalamic diaschisis in symptomatic cerebrovascular steno-occlusive patients. J Cereb Blood Flow Metab. 2020;40(3):563-573. https://doi.org/ 10.1177/0271678X19830532.

16. Kassner A, Winter JD, Poublanc J, Mikulis DJ, Crawley AP. Bloodoxygen level dependent MRI measures of cerebrovascular reactivity using a controlled respiratory challenge: reproducibility and gender differences. J Magn Reson Imaging. 2010;31(2):298-304.

17. Slessarev M, Han J, Mardimae A, Prisman E, Preiss D, Volgyesi G, et al. Prospective targeting and control of end-tidal $\mathrm{CO} 2$ and $\mathrm{O} 2$ concentrations. J Physiol. 2007;581(Pt 3):1207-19.

18. van Niftrik CHB, Piccirelli M, Bozinov O, Maldaner N, Strittmatter $\mathrm{C}$, Pangalu $\mathrm{A}$, et al. Impact of baseline $\mathrm{CO} 2$ on blood-oxygenationlevel-dependent MRI measurements of cerebrovascular reactivity and task-evoked signal activation. Magn Reson Imaging. 2018;49:123-30.

19. van Niftrik CHB, Piccirelli M, Bozinov O, Pangalu A, Fisher JA, Valavanis A, et al. Iterative analysis of cerebrovascular reactivity dynamic response by temporal decomposition. Brain Behav. 2017;7(9):e00705. https://doi.org/10.1002/brb3.705

20. Fischl B, Salat DH, van der Kouwe AJ, Makris N, Segonne F, Quinn BT, et al. Sequence-independent segmentation of magnetic resonance images. NeuroImage. 2004;23(Suppl 1):S69-84.

21. Dale AM, Fischl B, Sereno MI. Cortical surface-based analysis. I. Segmentation and surface reconstruction. NeuroImage. 1999;9(2): 179-94.

22. Baumgartner RW, Baumgartner I, Mattle HP, Schroth G. Transcranial color-coded duplex sonography in unilateral flowrestrictive extracranial carotid artery disease. AJNR Am J Neuroradiol. 1996;17(4):777-83.

23. Kultas-Ilinsky K, Ilinsky IA, Verney C. Glutamic acid decarboxylase isoform 65 immunoreactivity in the motor thalamus of humans and monkeys: gamma-aminobutyric acidergic connections and nuclear delineations. J Comp Neurol. 2011;519(14):2811-37.

24. Cicirata F, Zappala A, Serapide MF, Parenti R, Panto MR, Paz C. Different pontine projections to the two sides of the cerebellum. Brain Res Brain Res Rev. 2005;49(2):280-94.

25. Andrews RJ. Transhemispheric diaschisis. A review and comment. Stroke. 1991;22(7):943-9.

26. Gold L, Lauritzen M. Neuronal deactivation explains decreased cerebellar blood flow in response to focal cerebral ischemia or suppressed neocortical function. Proc Natl Acad Sci U S A. 2002;99(11):7699-704.

27. Carrera E, Tononi G. Diaschisis: past, present, future. Brain. 2014;137(Pt 9):2408-22.

Publisher's Note Springer Nature remains neutral with regard to jurisdictional claims in published maps and institutional affiliations. 